\title{
Adult Neurogenesis Is Regulated by Endogenous Factors Produced During Neurodegeneration
}

\author{
Masanori Yoneyama ${ }^{1}$, Tatsuo Shiba ${ }^{1}$, Shigeru Hasebe ${ }^{1}$, and Kiyokazu Ogita ${ }^{1, *}$ \\ ${ }^{1}$ Department of Pharmacology, Setsunan University Faculty of Pharmaceutical Sciences, \\ 45-1 Nagaotoge-cho, Hirakata, Osaka 573-0101, Japan
}

Received January 6, 2011; Accepted February 17, 2011

\begin{abstract}
Adult neurogenesis is the process of generating new neurons that become integrated into existing circuits after fetal and early postnatal development has ceased. In most mammalian species, adult neurogenesis only appears to occur in the olfactory bulb and the hippocampus, where neural stem/progenitor cells (NPCs) exist to create new neurons. In adult neurogenesis, microenviromental change is thought to provide a specific modulation for maintaining the multi-potent state of these NPCs. Neurodegeneration is driven by the activation of resident microglia, astrocytes, and infiltrating peripheral macrophages, which release a plethora of cytokines, chemokines, neurotransmitters, and reactive oxygen species. These endogenous factors cause further bystander damage to neurons and produces both detrimental and favorable conditions for neurogenesis. Interestingly, these endogenous factors also affect the proliferation, migration, differentiation, and survival of the NPCs, as well as regulate the incorporation of newly formed neurons into the brain circuitry. The unique profile of the endogenous factors released can vary the degree of neuroregeneration after neurodegeneration. This current review summarizes recent knowledge in the emerging field that is showing that adult neurogenesis is regulated by endogenous factors produced during neurodegeneration.
\end{abstract}

Keywords: microglia, neural stem/progenitor cells, neurodegeneration, neurogenesis

\section{Introduction}

A considerable large body of evidence suggests the prevailing view that neurogenesis occurs in the mature brain of adult mammals in addition to its occurrence in the developing brain. A part of the evidence for this view is the existence of neural stem/progenitor cells (NPCs) in the mammalian central nervous system. These NPCs are defined as primitive cells with self-renewal capacity and multi-potentially to generate several neural lineages including neurons, astrocytes, and oligodendrocytes. Although the NPCs are abundant in different discrete regions of the brain during embryonic and postnatal development, the adult brain contains these NPCs in limited brain regions including the subgranular zone

*Corresponding author. ogita@pharm.setsunan.ac.jp Published online in J-STAGE on March 16, 2011 (in advance) doi: 10.1254/jphs.11R02CP

Invited article
(SGZ) in the dentate gyrus and the subventricular zone (SVZ) in the lateral walls of the lateral ventricles, where the NPCs migrate and differentiate into neurons in the hippocampal dentate gyrus and the olfactory bulb, respectively (1). The NPCs in the SGZ are radial glia-like cells that are different from the cells of the SVZ. In the healthy adult brain, NPCs-mediated neurogenesis is involved in learning and memory and neural turnover in the hippocampal dentate gyrus and olfactory bulb. However, adult neurogenesis occurs not only primarily in the hippocampal dentate gyrus and olfactory bulb, but also in other areas throughout the brain. The evidence for this suggestion comes from the findings that NPCs exist in many discrete regions of the brain including the neocortex, spinal cord, tegmentum, substantia nigra, amygdala, and brain stem, as well as the SGZ and SVZ. Although the functional significance of the NPCs in these regions is unknown, such cells may await some stimulus to activate the process of neurogenesis.

In the 1990's, the "rediscovery" of neurogenesis was due in large part to the observation that the number of 
new neurons in the adult hippocampus is modulated by a range of factors, including stress, aging, environment, and locomotion activity. Numerous drugs and behaviors affect the number of new neurons in the brain (1). In addition, several neuropsychiatric conditions are associated with altered rates of neurogenesis in animal models such as Alzheimer's disease, temporal-lobe epilepsy, ischemia, and depression. In these cases, it remains unclear whether perturbed neurogenesis is a symptom of the disorder or has a causal role.

Although neurological deficits resulting from brain injury and neurodegenerative disorders are primarily due to neuronal death, the disruption of endogenous neurogenesis also contributes to neurological deficits as well as to hindering the recovery from brain insults. In the case of brain injury or neurodegenerative disorders, endogenous factors are released during neurodegeneration. The relationship between neuronal death and neurogenesis regulation has been documented but remains an object for intense investigation. Notably, recent collective reports indicate that during brain injury and in neurodegenerative disorders, neurogenesis is controlled by cytokines, chemokines, neurotransmitters, and reactive oxygen/nitrogen species (ROS), which are released by dying neurons as well as by activated macrophages, microglia, and astrocytes (2). This review provides an overview of recent findings showing that neurogenesis is regulated by endogenous factors including chemokines, cytokines, neurotransmitters, and ROS released from damaged neurons, microglia, and astrocytes under neuropathological conditions. Neurogenesis is completed by multiple steps including proliferation, migration, differentiation, survival, and integration of the newly formed neurons recruited into the circuitry of the brain. Finally, we summarize the effect of endogenous factors on these steps during neurogenesis.

\section{Brain injury}

Adult neurogenesis in the central nervous system during injury has been studied using animal models of neuronal damage and human patients with neurodegenerative disorders such as stroke, mechanical injury, Alzheimer's disease, Parkinson's disease, Huntington's disease, and amyotrophic lateral sclerosis.

\subsection{Stroke}

Brain ischemia enhances neurogenesis in the hippocampal dentate gyrus and SVZ of adult animals. The evidence for this enhancement comes from the finding that transient global ischemia in the brain increases the number of mitotic cells labeled with bromodeoxyuridine (BrdU) or expressing Ki67 in the hippocampal SGZ (3) and SVZ (4). BrdU-positive cells in the SGZ successfully migrate to the dentate granular cell layer, whereas those in the SVZ migrate to the olfactory bulb, cortex, and striatum. The majority of these BrdU-positive cells has been confirmed to differentiate into neurons in the respective regions.

\subsection{Mechanical damage}

Following mechanical damage, neurogenesis is not only facilitated in the hippocampus and olfactory bulb but also stimulated in the brain regions damaged by mechanical impact (5). Aspiration lesions of the sensorimotor cortex in adult rats produce a transient doubling of the number of cells in the SVZ with an increase in the expression of the immature neuronal marker known as polysialylated neural cell adhesion molecule (PSANCAM). In another experimental model of traumatic brain injury, BrdU-labeled cells increase the number in the SGZ at a maximal rate on day 3 post-injury (6). One month post-injury, these BrdU-labeled cells become recruited into the circuitry of the dentate gyrus as newly generated mature neurons, which are positive for calbindin. In an experimental murine model of severe compression spinal cord injury, proliferation, migration, and differentiation into mature neurons of endogenous NPCs in the spinal cord are increased at an early stage after the traumatic injury (7).

\subsection{Neurodegenerative disorders}

The studies on patients with Alzheimer's disease and a mouse model of Alzheimer's disease demonstrated that early neuronal differentiation markers, including TUC-4, doublecortin, and PSA-NCAM, are highly expressed in the hippocampus $(8,9)$. Evidence for NPC-generated dopaminergic projection neurons of the type that are lost in Parkinson's disease has been obtained by studying Parkinson's disease model mice prepared by treatment with 1-methyl-4-phenyl-1,2,3,6-tetrahydropyridine. These model mice show an increase in the number of BrdUlabeled proliferating cells within the substantia nigra post-injury (10). To investigate neurogenesis in the subependymal layer adjacent to the caudate nucleus in the human brain, a process that occurs in response to neurodegeneration of the caudate nucleus in Huntington's disease, postmortem control and Huntington's disease human brain tissues have been examined for the expression of proliferating cell nuclear antigen (PCNA), the cell cycle marker; $\beta$ III-tubulin, a neuronal marker; and glial fibrillary acidic protein (GFAP), an astrocyte marker. The results indicated a significant increase in cell proliferation in the subependymal layer in the Huntington-disease specimen compared with that in the control brains (11). In these experiments, PCNA-labeled 
cells were shown to co-express $\beta$ III-tubulin or GFAP, demonstrating the generation of neurons and glial cells in the subependymal layer of the diseased human brain. Further evidence for enhanced neurogenesis in Huntington's disease is also provided by studying the quinolinic acid-treated Huntington's disease model mice (12). Moreover, it has been demonstrated that amyotrophic lateral sclerosis-like transgenic mice show enhanced proliferative activity and vigorous migration of endogenous NPCs with differentiation into neurons within their spinal cord $(13,14)$.

\subsection{Neurotoxicant-induced brain injury}

An acute systemic treatment with kainic acid as an excitotoxin is classically known to produce limbic seizure and irreversible neuronal loss predominantly in pyramidal neurons of the hippocampal CA1 and CA3 in rats and mice. A large number of reports have provided evidence for enhanced neurogenesis in the dentate gyrus, although the functional significance of neurogenesis after pyramidal cell damage in the hippocampal CA regions remains to be elucidated. In addition to kainic acid, the organotin derivative trimethyltin (TMT) causes neuronal loss in the hippocampus $(15,16)$. In mice, in vivo acute systemic treatment with TMT produces severe loss of granule neurons in the dentate gyrus, without that of pyramidal neurons in the CA regions, on day 2 post-treatment. Our further observations have shown TMT-induced neuronal damage with a similar time course in the olfactory bulb of mice. Most interestingly, the granule cell layer is re- generated after severe loss of granule cells in the dentate gyrus in the TMT-treated mice. Thus, it is thought that the TMT-treated mice are useful as an animal model for evaluation of neuroregeneration through enhanced neurogenesis following neuronal damage in the dentate gurus and olfactory bulb, where neurogenesis mainly occurs under healthy conditions. Following TMT-induced granule loss in the dentate gyrus, the number of BrdUlabeled proliferating cells markedly increases on day $3-5$ in any of the dentate gyrus subregions including the SGZ, granule cell layer, hilus, and molecular layer (17). In addition to BrdU-labeled cells, nestin-positive cells also increase in number in all subregions of the dentate gyrus after TMT treatment (Fig. 1, upper panels). However, a TMT-induced increase in Ki67-labeled cells is observed predominantly in the SGZ and granule cell layer (Fig. 1, lower panels). These findings suggest the possibility that BrdU-labeled cells come from other regions, probably the SVZ, after TMT-induced granule cell loss. In addition to the dentate gyrus, the olfactory bulb also contains proliferating cells activated by neuronal damage induced by TMT treatment.

In TMT-treated mice, there are at least 3 phases for observation in the dentate gyrus after TMT treatment. These include the "neurodegeneration window" up to day 2, the "NPC proliferation window" from days 2 to 5 , and the "NPC migration/differentiation window." TMT treatment markedly activates microglia and astrocytes at the phase of NPC proliferation in the dentate gyrus. Table 1 shows the changes in the level of endogenous factors
Naive

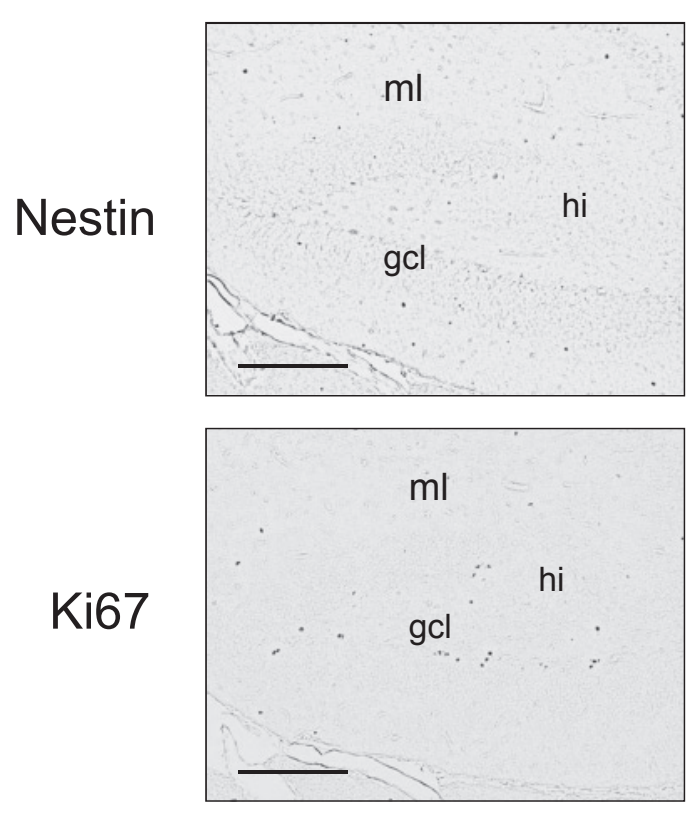

TMT
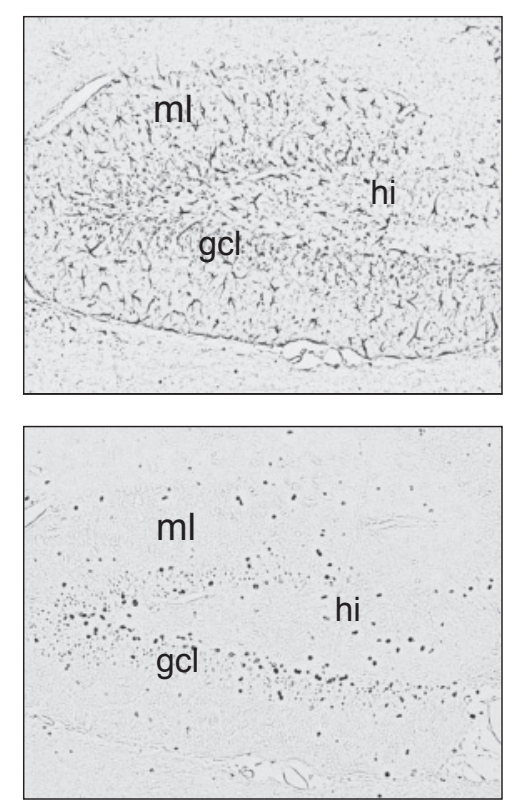

Fig. 1. Enhanced expression of nestin and Ki67 in the hippocampal dentate gyrus following granule cell loss treated with TMT. Mice were given TMT (2.8 $\mathrm{mg} / \mathrm{kg}$, i.p.) and then fixed on day 3 posttreatment for preparation of hippocampal sagittal sections, which were then subjected to immunostaining for Ki67 and nestin. Scale bar $=200 \mu \mathrm{m}$. gcl, granule cell layer; hi, hilus; ml, molecular layer. 
Table 1. Changes in gene expression in the hippocampus at an early step of neurogenesis following granule cell loss induced by TMT treatment

\begin{tabular}{lc}
\hline Genes & TMT $(\%$ of control $)$ \\
\hline TNF $\alpha$ & $576 \pm 124^{* *}$ \\
IL-1 $\beta$ & $259 \pm 84^{*}$ \\
IL-6 & $182 \pm 35^{*}$ \\
IFN $\gamma$ & $175 \pm 61$ \\
NOS1 (nNOS) & $70 \pm 34$ \\
NOS2 (iNOS) & $221 \pm 46^{*}$ \\
NOS3 (eNOS) & $86 \pm 10$ \\
STAT3 & $116 \pm 15$ \\
FasL & $87 \pm 25$ \\
Pax6 & $123 \pm 36^{*}$ \\
COX1 & $117 \pm 29$ \\
COX2 & $94 \pm 13$ \\
\hline
\end{tabular}

Mice were given TMT (2.8 mg/kg, i.p.) or saline and decapitated for preparation of total RNA on day 3 post-treatment. Total RNA was subjected to semi-quantitative RT-PCR for determining the expression level of the genes listed. $* P<0.05, * * P<0.01$, significantly different from the control value obtained for mice treated with saline. Values are from 4 separate animals.

released from activated microglia and astrocytes at the phase of NPC proliferation in the dentate gyrus of TMTtreated mice. TMT is capable of producing tumor necrosis factor $\alpha(\mathrm{TNF} \alpha)$, interleukin (IL)- $1 \beta$, and nitric oxide (NO) synthase (NOS)2 (iNOS) at the phase of NPC proliferation, but not during that of neurodegeneration, in the dentate gyrus. These observations suggest that these endogenous factors mediate neurogenesis in the dentate gyrus. Next we focus on endogenous factors released from glial cells activated by neurodegeneration.

\section{Pro-inflammatory cytokines}

Pro-inflammatory cytokines are produced predominantly by activated immune cells such as microglia and are involved in the amplification of inflammatory reactions. Such cytokines include IL-1, IL-6, and TNF $\alpha$. It is well established that inflammation plays a positive role in neurogenesis during brain ischemia. Multiple models of ischemic-induced inflammation have demonstrated an increase in neurogenesis (4). Alzheimer's disease is a chronic inflammatory disease that has been shown to cause elevated levels of neurogenesis. In addition to these disorders, TMT treated mice have the ability to enhance production of pro-inflammatory cytokines during the phase of NPC proliferation after neuronal damage.

\subsection{Interferon gamma (IFN $\gamma$ )}

Phenotypic changes in neurogenesis associated with inflammatory disease occur partially through the regulation and production of cytokines. Of several cytokines, IFN $\gamma$ promotes neural differentiation and neurite outgrowth in adult murine NPCs (18) and in the human neuroblastoma Paju cell line (19). However, IFN $\gamma$ has opposing dual effects on neurogenesis: one is stimulation of NPC migration and differentiation into neuronal cells and the other, inhibition of NPC proliferation and survival (20).

\subsection{TNF $\alpha$}

$\mathrm{TNF} \alpha$ is a pleiotropic pro-inflammatory cytokine that has been extensively investigated in neurodegenerative disorders. TNF $\alpha$ is predominantly expressed by activated microglia and, to a lesser extent, by astrocytes and neurons. Some reports indicated that TNF $\alpha$ has distinct effects on proliferation, differentiation, and migration in newly generated cells during neurogenesis process. $\mathrm{TNF} \alpha$ promotes the proliferation and differentiation into astrocytes in human fetal cortical NPCs (21). Another report demonstrated that TNF $\alpha$ is multifunctional, inhibiting NPC proliferation but stimulating migration of newborn rat striatal NPCs (20). Further studies will be needed to elucidate the functional significance of TNF $\alpha$ in neurogenesis.

\subsection{IL-1 $\beta$ and IL-6}

In vitro exposure of embryonic rat brain NPCs to IL$1 \beta$ inhibits their proliferation through apparent apoptosis induced by excess IL-1 $\beta$ (22). Conversely, others reported that IL-1 $\beta$ increases the proliferation of fetal human NPCs and inhibits their differentiation into neurons (21). IL-6 has the ability to cause NPCs to differentiate into astrocytes. In addition, transgenic mice with chronic expression of IL-6 have a dramatic reduction in hippocampal neurogenesis (23). It is thus thought that IL- $1 \beta$ and IL-6 work as gliogenesis factors, not as neurogenesis factors.

\section{Glutamate}

As the major excitatory neurotransmitter in the mammalian brain, glutamate can activate both fast excitatory synaptic potentials via ionotropic glutamate receptors and slow excitatory synaptic responses via metabotropic glutamate receptors (mGluRs). To date, 8 mGluR subtypes (mGluR1 - mGluR8) have been found in the mammalian brain and are classified into 3 groups with respect to their structural features, neuronal signaling, and pharmacological properties: Group I mGluRs include mGluR1 (with alternatively spliced variants, mGluR $1 \mathrm{a}-\mathrm{g}$ ) and 
mGluR5 (with differently spliced forms, mGluR5a and b); Group II mGluRs include mGluR2 and mGluR3; and Group III mGluRs include mGluR4 and mGluR6 - 8. The ionotropic glutamate receptors have been classified into 3 pharmacologically distinct groups: $\alpha$-amino-3hydroxy-5-methyl-4-isoxazole propionic acid (AMPA) receptors, kainate receptors, and NMDA receptors.

Endogenous factors released during the neurodegenerative process are not only pro-inflammatory cytokines expressed in damaged regions but also glutamate released from activated microglia and damaged neurons. Although an excess of extracellular glutamate is well-known to produce neuronal death, recent studies have shown the involvement of extracellular glutamate in proliferation, migration, and differentiation of the NPCs. Of the ionotropic glutamate receptors, NMDA receptors are involved in neurogenesis in both the developing and adult brains. As shown by in vitro studies, NMDA receptor-mediated activation of ERK and cAMP-response element binding protein promotes the proliferation of the NPCs derived from fetal cortex through an increase in intracellular $\mathrm{Ca}^{2+}$. In vivo experiments, however, revealed that treatment with NMDA-receptor antagonists significantly increases the number of newly generated neurons and radial glia-like cells in the dentate gyrus of the aged and young adult rats, suggesting that NMDA-receptor activation negatively regulates hippocampal neurogenesis (24). These paradoxical findings may due to the different experimental conditions between in vitro and in vivo experiments. To address these issues under different experimental conditions, we determined the effect of NMDA-receptor antagonists on the proliferation of proliferating cells in the hippocampal dentate gyrus of TMT-treated mice under both in vivo and in vitro experimental conditions (Fig. 2). As demonstrated above (Fig. 1), TMT treatment produced granule neuron loss ("neurodegeneration window"), with subsequent activation of the neurogenesis process for neuroregeneration ("NPC proliferation and migration/differentiation windows") in the dentate granule cell layer in mice. During the NPC proliferation window after TMT treatment in vivo, the NMDA-receptor antagonist MK-801 significantly decreased the number of BrdU-labeled cells from the SGZ (Fig. 2a). To prepare the NPCs in the dentate gyrus, we have tried to culture the proliferating cells derived from the dentate gyrus during the NPC proliferation window after TMT treatment in vivo. Finally, we succeeded in culturing NPC neurospheres, which were capable of differentiating into neurons, astrocytes, and oligodendrocytes, in the presence of growth factors and glutamate. In in vitro experiments using the cultured NPC neurospheres derived from the dentate gyrus of TMT-treated mice, exposure to MK-801 markedly inhibited the growth of the neurospheres in the presence of glutamate (Fig. 2b). Therefore, NMDA-receptor signals positively regulate neurogenesis in the dentate gyrus through activation of proliferative activity of the NPCs at least following neuronal degeneration.

mGluRs are important during development of the embryonic and adult brain. Whereas the blockage of mGluR5 or mGluR3 decreases cell proliferation and survival of cultured NPC neurospheres, the activation of mGluR5 increases cell proliferation of these neurospheres (25). In addition to the in vitro experiments, in vivo experiments have demonstrated that treatment with antagonists for mGluR3 or mGluR5S decreases proliferating cells in the SVZ and dentate gyrus of adult mice. Moreover, mGluR5-knockout mice have impaired learning and memory with a decrease in NPC proliferation (26). These findings all suggest that the activation of mGluR5 and mGluR3 are essential for neurogenesis in developing and adult brain. As a conclusion, glutamate released from microglia and neurons during neuronal degeneration activates NMDA receptors, mGluR3, and mGluR5 for promoting the proliferative activity of the NPCs.

\section{ROS}

ROS include the superoxide anion $\left(\cdot \mathrm{O}_{2}^{-}\right)$, hydrogen peroxide $\left(\mathrm{H}_{2} \mathrm{O}_{2}\right)$, hydroxyl radical $(\cdot \mathrm{HO}), \mathrm{NO}$, and peroxynitrite $\left(\mathrm{ONOO}^{-}\right)$. A large number of studies indicate that ROS-induced changes in the intracellular redox status influence cellular activities including signal transduction, metabolism, growth, and apoptosis, as well as cellular systems involved in detoxification. Particularly, NO has been implicated in various pathogenic conditions, such as inflammation, neurodegenerative disease, cardiovascular disease, and neoplasia. NO is produced by NOS, which exists as 3 different isoforms known as the neuronal isoform (NOS1, nNOS), inducible isoform (NOS2, iNOS), and endothelial isoform (NOS3, eNOS). NOS1 and NOS3 are activated by $\mathrm{Ca}^{2+}$ via interaction with calmodulin, whereas NOS2 is expressed in most types of infections caused by pathogens such as bacteria, protozoa, fungi, and viruses.

Accumulating evidence suggests that NO plays a critical role in neural development by affecting cell proliferation, migration, and differentiation. In neuronal cell lines and embryonic cells derived from neural tissues, NO attenuates cell proliferation and facilitates cell differentiation. Further evidence for the anti-proliferative effect of NO on NPCs comes from the observation that NOS inhibitors, for example, $N^{\omega}$-nitro-L-arginine methyl ester, significantly enhance neurogenesis in the SVZ and dentate gyrus of adult mice and rats (27). Contrariwise, NO is known to be essential for proliferation of glial tumor 
(a)

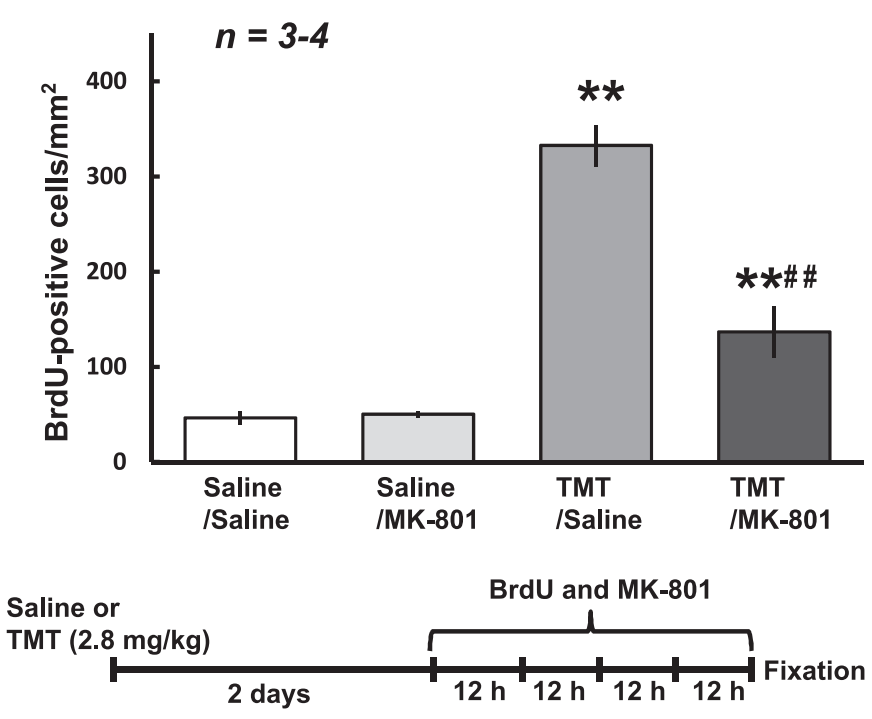

(b)

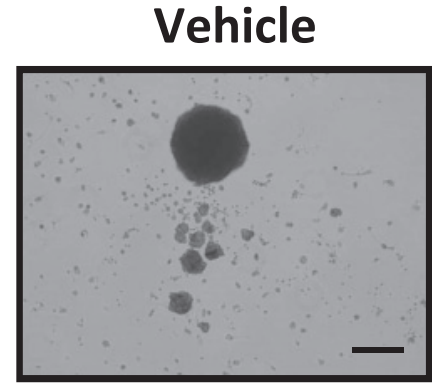

MK-801
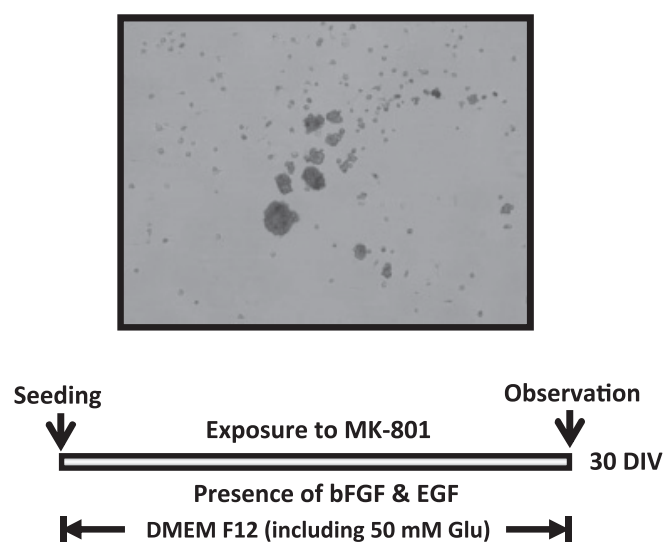

Fig. 2. Effect of MK801 on proliferation of the dentate NPCs generated following granule cell loss treated with TMT. a) Mice were given either saline or TMT (2.8 mg/kg, i.p.) and then treated with saline or MK-801 (0.5 mg/kg, s.c.) with BrdU (50 mg/kg, i.p.) every $12 \mathrm{~h}$ ( 4 times) from day 2 to 4 after TMT treatment. Following fixation at $12 \mathrm{~h}$ after the final injection of MK-801, brain coronal sections were immunostanined for BrdU. Values are expressed the mean \pm S.E.M. of BrdU-labeled cells in the SGZ. ${ }^{* *} P<0.01$, significantly different from the value obtained for animals treated with vehicle alone. ${ }^{\# \#} P<0.01$, significantly different from the value obtained for animals treated with TMT and saline. b) Cells were prepared from the hippocampal dentate gyrus of a mouse sacrificed on day 3 after TMT treatment and subsequently cultured in the DMEM F12 medium containing bFGF and EGF for 30 DIV in the absence or presence of MK-801. Under the conditions in the absence or presence of MK-801, round marked spheres were formed from the cells adhered to each other and subsequently proliferated to from large neurospheres in proportion to the duration of cultivation. Typical light micrographs of cells cultured for $30 \mathrm{DIV}$ after seeding. Scale bar $=200 \mu \mathrm{M}$. The cells are seeded in the DMEM F12 medium containing $50 \mu \mathrm{M}$ glutamate and $250 \mu \mathrm{M}$ glycine, which are concentrations sufficient for activation of NMDA receptors.

cell lines (28). Further evidence for the essential role of endogenous NO and other ROS in the proliferation of NPCs comes from our recent findings that inhibitors of NOS and NADPH oxidase have the ability to attenuate proliferation of the NPCs derived from the hippocampus of embryonic mice (29).

The finding that enhanced expression of NOS2 (iNOS) after focal cerebral ischemia stimulates neurogenesis in the dentate gyrus supports the proposition that NO enhances neurogenesis in the dentate gyrus. In nNOSknockout mice, however, cerebral ischemia-induced NPC proliferation and survival of newly formed neurons is enhanced in the dentate gyrus (30). Since the focal cerebral ischemia produces an increase in the level of iNOS as well as a decrease in nNOS level in the hippocampus, it is thought that enhancement of neurogenesis following brain ischemia may be due to up-regulation of iNOS after the decrease in nNOS in the hippocampus. Under neuro-inflammatory conditions such as brain ischemia, down-regulation of nNOS and up-regulation of iNOS may thus act as a mechanism underlying enhancement of neurogenesis for recovery from the neuronal degeneration.

\section{Conclusions}

In this present review article, we documented that following neuronal damage in the brain, neurogenesis is promoted in the damaged portion of the brain through enhanced proliferation, differentiation, and migration of endogenous NPCs existing in the hippocampal SGZ and SVZ. The neurogenesis is positively regulated by numer- 
Table 2. Effects of endogenous factors and enzymes on proliferation, differentiation, and migration of NPCs during neurogenesis

\begin{tabular}{lcccc}
\hline $\begin{array}{l}\text { Endogenous } \\
\text { factors/enzymes }\end{array}$ & Proliferation & Differentiation & Migration & References \\
\hline IFN $\gamma$ & $\downarrow$ & $\uparrow$ & $\uparrow$ & $18-20$ \\
TNF $\alpha$ & $\uparrow$ or $\downarrow$ & $\downarrow$ or $\uparrow$ & $\uparrow$ & 20,21 \\
IL-1 $\beta$ & $\uparrow$ or $\downarrow$ & - & - & 22 \\
IL-6 & $\downarrow$ & $\downarrow$ & - & 23 \\
Glutamate & $\uparrow$ or $\downarrow$ & $\uparrow$ & - & $24-26$ \\
NOS1 (nNOS) & $\downarrow$ & $\uparrow$ & - & 27,30 \\
NOS2 (eNOS) & $\uparrow$ & $\uparrow$ & - & 28 \\
\hline
\end{tabular}

$\uparrow$, enhancement ; $\downarrow$, attenuation ; -, no report.

ous endogenous factors, such as pro-inflammatory factors, glutamate, and ROS, that are released from microglia and astrocytes activated after neuronal damage (Table 2). Further studies will be needed to evaluate the mechanisms underlying the regulation of neurogenesis by these factors in the damaged portion of the brain. Such future studies would hopefully contribute to the development of therapeutics for neurodegenerative disorders.

Two therapeutic strategies to enhance neurogenesis currently exist. The first is transplantation of exogenous NPCs. Exogenous NPCs for transplantation can be isolated from electively aborted fetuses or derived from embryonic stem/progenitor cell lines. However, these strategies face many ethical and host-versus-donor rejection issues. Recent studies have shown that stem cells derived from non-neural tissues, including skin, bone marrow, and adipose tissue, can be trans-differentiated into neural stem cells. These non-neuronal-derived NPCs transplanted directly into the brain or introduced by intravenous injection would be expected to repopulate the damaged portion of the brain. The use of patient' NPCs trans-differentiated from their own non-neuronal stem cells to create new self-neurons in individuals would remove the probably of host-versus-donor rejection. A second strategy would be to stimulate the proliferation, migration, and differentiation of endogenous NPCs. A full understanding of mechanisms underlying neurogenesis following neurodegeneration should contribute to the development of therapeutic drugs for neuronal regeneration through enhancement of neurogenesis in the damaged portion of the brain.

\section{References}

1 Ming GL, Song H. Adult neurogenesis in the mammalian central nervous system. Annu Rev Neurosci. 2005;28:223-250.

2 Whitney NP, Eidem TM, Peng H, Huang Y, Zheng JC. Inflamma- tion mediates varying effects in neurogenesis: Relevance to the pathogenesis of brain injury and neurodegenerative disorders. $\mathrm{J}$ Neurochem. 2009;108:1343-1359.

3 Darsalia V, Heldmann U, Lindvall O, Kokaia Z. Stroke-induced neurogenesis in aged brain. Stroke. 2005;36:1790-1795.

4 Tang H, Wang Y, Xie L, Mao X, Won SJ, Galvan V, Jin K. Effect of neural precursor proliferation level on neurogenesis in rat brain during aging and after focal ischemia. Neurobiol Aging. 2009;30:299-308.

5 Parent JM. Injury-induced neurogenesis in the adult mammalian brain. Neuroscientist. 2003;9:261-272.

6 Dash PK, Mach SA, Moore AN. Enhanced neurogenesis in the rodent hippocampus following traumatic brain injury. J Neurosci Res. 2001;63:313-319.

7 Ke Y, Chi L, Xu R, Luo C, Gozal D, Liu R. Early response of endogenous adult neural progenitor cells to acute spinal cord injury in mice. Stem Cells. 2006;24:1011-1019.

8 Jin K, Galvan V, Xie L, Mao XO, Gorostiza OF, Bredesen DE, et al. Enhanced neurogenesis in Alzheimer's disease transgenic (PDGF-APPSw, Ind) mice. Proc Natl Acad Sci U S A. 2004; 101:13363-13367.

9 Jin K, Peel AL, Mao XO, Xie L, Cottrell BA, Henshall DC, et al. Increased hippocampal neurogenesis in Alzheimer's disease. Proc Natl Acad Sci U S A. 2004;101:343-347.

10 Zhao M, Momma S, Delfani K, Carlen M, Cassidy RM, Johansson $\mathrm{CB}$, et al. Evidence for neurogenesis in the adult mammalian substantia nigra. Proc Natl Acad Sci U S A. 2003; 100:7925-7930.

11 Curtis MA, Penney EB, Pearson AG, van Roon-Mom WM, Butterworth NJ, Dragunow M, et al. Increased cell proliferation and neurogenesis in the adult human Huntington's disease brain. Proc Natl Acad Sci U S A. 2003;100:9023-9027.

12 Tattersfield AS, Croon RJ, Liu YW, Kells AP, Faull RL, Connor B. Neurogenesis in the striatum of the quinolinic acid lesion model of Huntington's disease. Neuroscience. 2004;127:319 332.

13 Chi L, Gan L, Luo C, Lien L, Liu R. Temporal response of neural progenitor cells to disease onset and progression in amyotrophic lateral sclerosis-like transgenic mice. Stem Cells Dev. 2007;16: 579-588.

14 Corti S, Locatelli F, Papadimitriou D, Del Bo R, Nizzardo M, Nardini M, et al. Neural stem cells LewisX+ CXCR4+ modify 
disease progression in an amyotrophic lateral sclerosis model. Brain. 2007;130:1289-1305.

15 Shuto M, Higuchi K, Sugiyama C, Yoneyama M, Kuramoto K, Nagashima R, et al. Endogenous and exogenous glucocorticoids prevent trimethyltin from causing neuronal degeneration of the mouse brain in vivo: involvement of oxidative stress pathways. $\mathrm{J}$ Pharmacol Sci. 2009;110:424-436.

16 Shuto M, Seko K, Kuramoto N, Sugiyama C, Kawada K, Yoneyama M, et al. Activation of c-Jun N-terminal kinase cascades is involved in part of the neuronal degeneration induced by trimethyltin in cortical neurons of mice. J Pharmacol Sci. 2009; 109:60-70.

17 Ogita K, Nishiyama N, Sugiyama C, Higuchi K, Yoneyama M, Yoneda Y. Regeneration of granule neurons after lesioning of hippocampal dentate gyrus: evaluation using adult mice treated with trimethyltin chloride as a model. J Neurosci Res. 2005; 82:609-621.

18 Wong G, Goldshmit Y, Turnley AM. Interferon-gamma but not TNF alpha promotes neuronal differentiation and neurite outgrowth of murine adult neural stem cells. Exp Neurol. 2004; 187:171-177.

19 Song JH, Wang CX, Song DK, Wangx P, Shuaib A, Hao C. Interferon gamma induces neurite outgrowth by up-regulation of $\mathrm{p} 35$ neuron-specific cyclin-dependent kinase 5 activation of ERK1/2 pathway. J Biol Chem. 2005;280:12896-12901.

20 Ben-Hur T, Ben-Menachem O, Furer V, Einstein O, Mizrachi-Kol $\mathrm{R}$, Grigoriadis N. Effects of proinflammatory cytokines on the growth, fate, and motility of multipotential neural precursor cells. Mol Cell Neurosci. 2003;24:623-631.

21 Peng H, Whittney N, Wu Y, Tian C, Dou H, Zhou Y, et al. HIV-1infected and/or immune-activated macrophage-secreted TNFalpha affects human fetal cortical neural progenitor cell proliferation and differentiation. Glia. 2008;56:903-916.

22 Wang X, Fu S, Wang Y, Yu P, Hu J, Gu W, et al. Interleukin-1 beta mediates proliferation and differentiation of multipotent neural precursor cells through the activation of SPAK/JNK pathway. Mol Cell Neurosci. 2007;36:343-354.

23 Vallieres L, Campbell IL, Gage FH, Sawchenko PE. Reduced hippocampal neurogenesis in adult transgenic mice with chronic astrocytic production of interleukin-6. J Neurosci. 2002;22: 486-492.

24 Nacher J, Alonso-L losa G, Rosell DR, McEwen BS. MNDA receptor antagonist treatment increase the production of new neurons in the aged rat hippocampus. Neurobiol Aging. 2003;24: 273-284.

25 Di Giorgi-Gerevini V, Melchiorri D, Battaglia G, Ricci-Vitiani L, Ciceroni C, Busceti CL, et al. Endogenous activation of metabotropic glutamate receptors supports the proliferation and survival of neural progenitor cells. Cell Death Differ. 2005; 12:1124-1133.

26 Kinney GG, Burno M, Campbell UC, Hernandez LM, Rodriguez D, Bristow LJ, et al. Metabotropic glutamate subtype 5 receptors modulate locomotor activity and sensorimotor gating in rodents. J Pharmacol Exp Ther. 2003;306:116-123.

27 Romero-Grimaldi C, Gheusi G, Lledo PM, Estrada C. Chronic inhibition of nitric oxide synthesis enhances both subventricular zone neurogenesis and olfactory learning in adult mice. Eur J Neurosci. 2006;24:2461-2470.

28 Tanriover N, Ulu MO, Isler C, Durak H, Oz B, Uzan M, et al. Neuronal nitric oxide synthase expression in glial tumors: correlation with malignancy and tumor proliferation. Neurol Res. 2008;30:940-944.

29 Yoneyama M, Kawada K, Gotoh Y, Shiba T, Ogita K. Endogenous reactive oxygen species are essential for proliferation of neural stem/progenitor cells. Neurochem Int. 2010;56:740-746.

30 Fritzen S, Schmitt A, Koth K, Sommer C, Lesch KP, Reif A. Neuronal nitric oxide synthase (NOS-I) knockout increases the survival rate of neural cells in the hippocampus independently of BDNF. Mol Cell Neurosci. 2007;35:261-271. 